Pacific Journal of Mathematics

MEASURES ON COUNTABLE PRODUCT SPACES 


\title{
MEASURES ON COUNTABLE PRODUCT SPACES
}

\author{
E. O. Elliott
}

A regular conditional measure $\nu$ on a space $Y$ relative to an outer measure $\mu$ on a space $X$ is defined as a function on $X \times \mathscr{R}$ such that $(1)$ for each $x \in X, \nu(x, \cdot)$ is an outer measure on $Y$ and $\mathscr{R}$ is the family of subsets of $Y$ which are (Carathéodory) measurable under each of the measures $\nu(x, \cdot), x \in X$, and $(2)$ for each $\beta \in \mathscr{R}$ the function $\nu(\cdot, \beta)$ on $X$ is $\mu$ integrable $)$ i.e., $\left.\int \nu(x, \beta) \mu d x \leqq \infty\right)$.

Letting $g$ be the function on the subsets of $Z=X \times Y$ defined by

$$
g(\beta)=\iint I_{\beta}(x, y) \nu(x, \cdot) d y \mu d x,
$$

defining a covering family $\Im$ to consist of those rectangles $A \times B$ where $A$ is $\mu$ measurable, $B \in \mathscr{R}$ and $g(A \times B)<\infty$ or those sets $N$ such that $g(N)=0$, we obtain the outer measure $\phi=(\mu \circ \nu)$ on $Z$ generated by (the content) $g$ and covering family $\mathfrak{s}$.

A system of regular conditional measures is a sequence begun by a measure $\nu_{0}$ on a space $X_{1}$ and followed by regular conditional measures $\nu_{i}\left(\right.$ relative to $\left.\mu_{i}\right)$ on spaces $X_{i+1}(i=1,2, \cdots)$ where $\mu_{1}=\nu_{0}$ and $\mu_{i+1}=\left(\mu_{i} \circ \nu_{i}\right)$ for $i=1,2, \cdots$. Set $X=$ $\Pi_{i} X_{i}$, and for $x \in X$ write $x^{i}$ for the point $\left(x_{1}, x_{2}, \cdots, x_{i}\right)$ which is the projection of $x$ onto the space $X^{i}=\prod_{j=1}^{i} X_{j}$ and similarly write $S^{i}=\prod_{j=1}^{i} S_{j}$ whenever the sets $S_{j}$ are subsets of $X_{j}(j=1, \cdots, i)$.

For such a system of regular conditional measures a generalization of Tulcea's extension theorem for regular conditional probabilities holds, a Fubini-like theorem for integrable functions is obtained and finally, for topological spaces, a condition is given for the extension of inner regularity and almost Lindelöfness properties.

We let $\mathscr{R}_{1}$ be the family of $\nu_{0}$ measurable sets and let $\mathscr{R}_{i}$ be the family of subsets of $X_{i}$ which are measurable under each of the measures $\nu_{i-1}\left(x^{i-1}, \cdot\right), x^{i-1} \in X^{i-1}$, and let $\Im_{i}$ be the family of subsets $\gamma$ of $X^{i}$ such that $\mu_{i}(\gamma)=0$ or $\gamma=\alpha \times \beta$ where $\alpha$ is $\mu_{i-1}$ measurable and $\beta \in \mathscr{R}_{i}$ and $\mu_{i}(\gamma)<\infty$. Thus $\mathfrak{\Im}_{i}$ is the covering family which generates $\mu_{i}$.

Now, writing $X_{i}^{*}=\prod_{j=i+1}^{\infty} X_{j}$ we define

$$
\begin{aligned}
\mathscr{R}^{*} & =\left\{S: S=\prod_{i} \beta_{i} \text { for some } \beta \text { s.t. } \beta_{i} \in \mathscr{R}_{i} \text { for each } i\right\} \\
\mathscr{R}^{* *} & =\left\{\beta: \text { for some } i, \beta=\alpha \times X_{i}^{*} \text { where } \alpha \subset X^{i} \text { and } \mu_{i}(\alpha)=0\right\}, \\
\mathscr{R} & =\mathscr{R}^{*} \cup \mathscr{R}^{* *},
\end{aligned}
$$


$g$ to be the function on $\mathscr{R}$ which is zero on $\mathscr{R}^{* *}$ and given by

$$
g(\beta)=\lim _{i} \mu_{i}\left(\beta^{i}\right)
$$

on $\mathscr{R}^{*}$.

For $\beta \in \mathscr{R}^{*}$ and $x \in X$, let

$$
\rho_{i}(x, \beta)=\nu_{0}\left(\beta_{1}\right) \prod_{j=1}^{i-1} \nu_{j}\left(x^{j}, \beta_{j+1}\right),
$$

and

$$
\rho(x, \beta)=\lim _{i} \rho_{i}(x, \beta) .
$$

Let $\mathscr{R}^{* \prime}=\left\{\beta \in \mathscr{R}^{*}: g(\beta)<\infty, \rho_{i}(x, \beta)\right.$ is uniformly bounded on $\beta$, and $\rho(x, \beta)$ exists for all $x \in \beta\}$ and $\mathscr{R}^{\prime}=\mathscr{R}^{* \prime} \cup \mathscr{R}^{* *}$ and use $\mathscr{R}^{\prime}$ and $g$ to generate a measure $\varphi$ on $X$.

Our first objectives are to prove that $\varphi$ and $g$ agree on the covering family $\mathscr{R}^{\prime}$ and that members of $\mathscr{R}$ are $\phi$ measurable. To do this we need and state a generalization of Tulcea's extension theorem for regular conditional probabilities. The final objective is to show that the product topology on $X$ is inner regular and almost Lindelöf [1] whenever the component spaces are provided the spaces are of finite measure and the conditional measures are continuous [1]. The proof of this parallels that given for general product measures [2].

1. A generalization of Tulcea's extension theorem. Let a regular conditional measure system $\nu_{i}^{\prime}$ be given as above and assume that $\nu_{v}^{\prime}\left(X_{1}\right)=1$ and $\nu_{i}^{\prime}\left(x^{i}, X_{i+1}\right)=1$ for each $i$ and $x^{i} \in X^{i}$, i.e., $\nu_{i}^{\prime}$ is a system of regular conditional probabilities. Define the measures $\mu_{i}^{\prime}$ as above with $\mu_{1}^{\prime}=\nu_{0}^{\prime}$ and $\mu_{i+1}^{\prime}=\left(\mu_{i}^{\prime} \circ \nu_{i}^{\prime}\right)$ and let $\mathscr{C}$ be the family of subsets of $X$ which are cylinders in $X$ over sets which are $\mu_{i}^{\prime}$ measurable for some $i$.

Now let $\Psi$ be the measure on $X$ generated by the covering family $\mathscr{K}$ and the content $h$ defined by

$$
h(\beta)=\mu_{2}^{\prime}(\alpha)
$$

where $\alpha \subset X^{\imath}$ and $\beta=\alpha \times X_{i}^{*} \in \mathscr{K}$.

The measure $\Psi$ differs from the conventional Tulcea extension of the conditional probabilities $\nu_{i}^{\prime}$ in that in going from $\mu_{i}^{\prime}$ to $\mu_{i+1}^{\prime}$ the sets $\alpha \subset X^{i+1}$ for which

$$
\iint I_{\alpha}\left(x^{i+1}\right) \nu_{i}^{\prime}\left(x^{i}, \cdot\right) d x_{i+1} \mu_{i}^{\prime} d x^{i}=0
$$

are assigned measure zero whereas, in the conventional extension they may not even be measurable. The conventional method of proof [3] 
for Tulcea's extension theorem, however, may be carried through for this new extension with essentially no changes. Therefore we give without proof the following.

THEOREM 1.1. The members of $\mathscr{K}$ are $\Psi$ measurable and $\Psi(\beta)=$ $h(\beta)$ for each $\beta \in \mathscr{K}$.

2. Agreement and measurability. Consider a member $S$ of $\mathscr{R}^{* \prime}$ and let $\nu_{0}^{\prime}(\cdot)=\nu_{0}(\cdot) / \nu_{0}\left(S_{1}\right)$ to get a normalized measure on $S_{1}$, and let $\nu_{i}^{\prime}\left(x^{i}, \cdot\right)=\nu\left(x^{i}, \cdot\right) / \nu_{i}\left(x^{i}, S_{i+1}\right)$ to get a regular conditional probability on $S_{i+1}$. Extending this system of probabilities as in $\$ 1$ yields a (probability) measure $\Psi_{S}$ on the space $S$. Let the measures $\mu_{i}^{\prime}$ on $S^{i}$ be associated with the $\nu_{i}^{\prime}$ as in $\$ 1$.

If $\beta \in \mathscr{R}^{* \prime}$ and $\beta \subset S$ then $\mu_{i}\left(\beta^{i}\right)$ is given by an $i$ fold integral in

$$
\begin{aligned}
\mu_{i}\left(\beta^{i}\right) & =\int(i) \int I_{\beta i}\left(x^{i}\right) \nu_{i-1}\left(x^{i-1}, \cdot\right) d x_{i} \cdots \nu_{1}\left(x^{1}, \cdot\right) d x_{2} \nu_{0} d x_{1} \\
& =\int(i) \int I_{\beta}\left(x^{i}\right) \rho_{i}(x, S) \nu_{i-1}^{\prime}\left(x^{i-1}, \cdot\right) d x_{i} \cdots \nu_{1}^{\prime}\left(x^{1}, \cdot\right) d x_{2} \nu_{0}^{\prime} d x_{1} \\
& =\int I_{\beta i}\left(x^{i}\right) \rho_{i}(x, S) \mu_{i}^{\prime} d x^{i} \\
& =\int I_{\beta i}\left(x^{i}\right) \rho_{i}(x, S) \Psi_{S} d x .
\end{aligned}
$$

Thus, employing Lebesgue's theorem, we have

$$
\begin{aligned}
g(\beta) & =\lim _{i} \mu_{i}\left(\beta^{i}\right) \\
& =\lim _{i} \int I_{\beta}\left(x^{i}\right) \rho_{i}(x, S) \Psi_{S} d x \\
& =\int \lim _{i} I_{\beta i}\left(x^{i}\right) \rho_{i}(x, S) \Psi_{S} d x \\
& =\int I_{\beta}(x) \rho(x, S) \Psi_{S} d x .
\end{aligned}
$$

Suppose now that $\mathscr{G} \subset \mathscr{R}^{\prime}, \mathscr{G}$ is countable, and $S=\cup \mathscr{G}$, then the members of $\mathscr{G}$ are $\Psi_{S}$ measurable since the members of $\mathscr{G}_{1}=\mathscr{G} \cap \mathscr{R}^{* \prime}$ are countable intersections of members of $\mathscr{K}$ (i.e., cylinders over $\mu_{2}^{\prime}$ measurable sets for some $i$ ) and members of $\mathscr{G}_{2}=\mathscr{G} \cap \mathscr{R}^{* *}$ have $\Psi_{S}$ measure zero. Hence,

$$
I_{S}(x) \leqq \sum_{\beta \in \mathscr{G}} I_{\beta}(x)
$$

and

$$
I_{S}(x) \leqq \sum_{\beta \in \mathscr{G}_{1}} I_{\beta}(x) \quad \text { a.e. } \Psi_{S}
$$


Consequently,

$$
\int I_{S}(x) \rho(x, S) \Psi_{S} d x \leqq \sum_{\beta \in \mathscr{\Phi}_{1}} \int I_{\beta}(x) \rho(x, S) \Psi_{S} d x+0
$$

and

$$
\begin{aligned}
g(S) & \leqq \sum_{\beta \in \mathscr{C}_{1}} g(\beta)+0 \\
& \leqq \sum_{\beta \in \mathscr{G}} g(\beta),
\end{aligned}
$$

and we conclude $g(S)=\varphi(S)$ proving the

THeOREM 2.1. If $S \in \mathscr{R}^{\prime}$ then $\varphi(S)=g(S)$. Let

$$
\mathscr{C l}=\left\{A: A=X^{i-1} \times \beta_{i} \times X_{i}^{*} \text { for some } i \text { and } \beta_{i} \in \mathscr{R}_{i}\right\}
$$

and note that if $A \in \mathscr{M l}$ and $S \in \mathscr{R}^{\prime}$ then

$$
S \cap A \in \mathscr{R}^{\prime} \text { and } S-A \in \mathscr{R}^{\prime}
$$

and

$$
\varphi(S)=\varphi(S \cap A)+\varphi(S-A) .
$$

We consequently learn that members of $\mathscr{C}$ are $\phi$ measurable since $\mathscr{R}^{\prime}$ is the covering family for $\varphi$. Countable intersections of members of $\mathscr{C}$ are hence measurable proving the next

Theorem 2.2. If $\beta \in \mathscr{R}$ then $\beta$ is $\varphi$ measurable.

For $x^{i} \in X^{i}$ let $\xi_{0}(\cdot)=\nu_{i}\left(x^{i}, \cdot\right)$, write $x^{i} y^{j}$ for the point $\left(x_{1}, \cdots, x_{i}\right.$, $\left.y_{1}, \cdots, y_{j}\right)$ and let $\xi_{j}\left(y^{j}, \cdot\right)=\nu_{i}\left(x^{i} y^{j}, \cdot\right), j=1,2, \cdots$. The regular conditional measure system $\xi_{j}$ then determines a measure $\lambda_{i}\left(x^{i}, \cdot\right)$ on $X_{i}^{*}$. For $\beta \subset X$ let us agree that $\beta_{x} i=\left\{y:\left(x_{1}, \cdots, x_{i}, y_{1}, y_{2}, \cdots\right) \in \beta\right\}$. Then we may state the

THEOREM 2.3. If $\beta$ is $\phi$ measurable then

$$
\varphi(\beta)=\int \lambda_{i}\left(x^{i}, \beta_{x} i\right) \mu_{i} d x^{i}=\iint I_{\beta}\left(x^{i} y\right) \lambda_{i}\left(x^{i}, \cdot\right) d y \mu_{i} d x^{i}
$$

and $\lambda_{i}$ is a regular conditional measure associated with $\mu_{i}$.

From [1, 1.6] we obtain the Fubini-like

THEOREM 2.4. If $f$ is $\varphi$ integrable $e^{1}$ then

$1-\infty \leqq \int f(z) \varphi d z \leqq \infty$ and $\{z: f(z) \neq 0\}$ is $\sigma$-finite under $\varphi$. 


$$
\int f(z) \varphi d z=\iint f\left(x^{i}, y\right) \lambda_{i}\left(x^{i}, \cdot\right) d y \mu_{i} d x^{i}
$$

3. Topological measures. To review the topological notions in [1] let us suppose that $T$ is a topological space with $\mathscr{T}$ being its family of open sets, and let $\theta$ be a measure on $T$ for which the open sets are measurable. Then $\mathscr{T}$ is almost Lindelöf (a.L.) provided each covering of $T$ by open sets contains a countable subfamily which covers almost all of $T$, and $\mathscr{T}$ is inner regular (i.r.) provided each open set can be approximated in measure by closed subsets of finite measure, i.e., for each $\beta \in \mathscr{T}$,

$$
\theta(\beta)=\operatorname{Sup}_{\gamma} \underset{\gamma \text { closed }}{\subset \beta,} \theta(\gamma)<\infty .
$$

Now let us assume that each of the spaces $X_{i}$ is endowed with a topology $\mathscr{T}_{i}$ and that $\mathscr{T}^{i}$ is the product of the topologies $\mathscr{T}_{j}$, $1 \leqq j \leqq i$. Then the sequence $\mathscr{T}_{i}$ will be called a.L. and i.r. provided $\mathscr{G}_{1}$ is a.L. and i.r. relative to $\nu_{0}$ and $\mathscr{T}_{i}$ is a.L. and i.r. relative to $\nu_{i-1}\left(x^{i-1}, \cdot\right)$ for each $x^{i-1} \in X^{i-1}$, and the sequence $\nu_{i}$ will be called continuous provided that for each $i=1,2, \cdots$, the function $\nu_{i}(\cdot, \beta)$ is finite and $\mathscr{T}^{i}$ continuous for each set $\beta$ which is measurable under all measures $\nu_{i}\left(x^{i}, \cdot\right)$ where $x^{i} \in X^{i}$.

From [1,2.3] and mathematical induction we obtain the

THEorem 3.1. If $\mathscr{T}_{i}$ is a.L. and i.r., $\nu_{i}$ is continuous and $\mu_{i}\left(X^{i}\right)<\infty$ for each $i$, then $\mathscr{T}^{i}$ is a.L. and i.r. relative to $\mu_{i}$ for each $i$.

Let $\mathscr{T}$ be the product topology on $X$ obtained from the $\mathscr{F}_{i}$. Then we have the

THeOREM 3.2. If $\mathscr{T}_{i}$ is a.L. and i.r., $\nu_{i}$ is continuous, $\mu_{i}\left(X^{i}\right)<\infty$ for each $i$, and $\varphi(X)<\infty$ then $\mathscr{T}$ is a.L. and i.r. relative to $\varphi$.

Proof. Suppose $A \in \mathscr{T}$, then for some countable family $\mathscr{C}$ such that each $\alpha \in \mathscr{C}$ is a cylinder $\alpha^{\prime} \times \alpha^{\prime \prime}$ where $\alpha^{\prime} \in \mathscr{T}^{i(\alpha)}$ and $\alpha^{\prime \prime}=X_{i}^{*}(\alpha)$ we have $A=\cup \mathscr{C}$. Since $\alpha^{\prime}$ above is $\mu_{i(\alpha)}$ measurable, $\alpha$ is $\varphi$ measurable and consequently $A$ is $\phi$ measurable. Since $\varphi(X)<\infty$ and each set $\alpha^{\prime}$ can be $\mu_{i(\alpha)}$ approximated by a closed subset as closely as desired, it follows that each $\alpha \in \mathscr{O}$ can be $\varphi$ approximated as closely as desired by the (closed) cylinders over those closed subsets. Since $\varphi(A)<\infty$ a finite subfamily $\mathscr{C}^{\prime}$ of $\mathscr{C}$ can be chosen so that $\varphi\left(\cup \mathscr{C}^{\prime}\right)$ is as close to $\varphi(A)$ as desired. Hence $A$ may be $\varphi$ approximated as closely as desired by closed subsets (which are the union of the closed cylinders 
associated with $\mathscr{C}^{\prime}$ ). Thus $\mathscr{T}$ is i.r. relative to $\varphi$.

To see that $\mathscr{T}$ is a.L., let $\mathscr{C}$ be an open covering of $X$ and let $\mathscr{H}_{i}$ be the family of open sets in $X^{i}$ such that each cylinder in $X$ over one of these open sets is a subset of some member of $\mathscr{H}$. Thus, letting

$$
\mathscr{C}_{i}=\left\{\beta: \beta=\alpha \times X_{i}^{*} \text { for some } \alpha \in \mathscr{C}_{i}\right\}
$$

we see that members of $\mathscr{C}_{i}$ belong to the base for the topology $\mathscr{T}$ and that $X=\mathbf{U} \mathscr{C}=\mathbf{U}_{i} \cup \mathscr{C}_{i}$. Using the fact that $\mathscr{T}^{i}$ is both i.r. and a.L. we can select a countable subfamily $\mathscr{H}_{i}^{\prime}$ of $\mathscr{H}_{i}$ for which $\mu_{i}\left(\bigcup \mathscr{C}_{i}-\bigcup \mathscr{C}_{i}^{\prime}\right)=0$. Now, letting

$$
\mathscr{C}_{i}^{\prime}=\left\{\beta: \beta=\alpha \times X_{i}^{*} \text { for some } \alpha \in \mathscr{C}_{i}^{\prime}\right\}
$$

we have $\phi\left(\mathbf{U} \mathscr{C}_{i}-\mathbf{U} \mathscr{C}_{i}^{\prime}\right)=0$ and taking $\mathscr{B}_{i}$ to be such a countable subfamily of $\mathscr{C}$ that each member of $\mathscr{C}_{i}^{\prime}$ is a subset of some member of $\mathscr{B}_{i}$, we obtain further that $\phi\left(\bigcup \mathscr{C}_{i}-\mathbf{U} \mathscr{B}_{i}\right)=0$.

Finally, let $\mathscr{B}=\bigcup_{i} \mathscr{B} \mathscr{P}_{i}$ and conclude,

$$
\begin{aligned}
X-\bigcup \mathscr{B}= & \bigcup_{i} \bigcup \mathscr{C}_{i}-\bigcup_{i} \bigcup \mathscr{B}_{i} \\
& \subset \bigcup_{i}\left(\mathbf{U} \mathscr{C}_{i}-\mathbf{U} \mathscr{B}_{i}\right)
\end{aligned}
$$

and

$$
\phi(X-\bigcup \mathscr{B}) \leqq \sum_{i} \phi\left(\mathbf{U} \mathscr{C}_{i}-\mathbf{U} \mathscr{P}_{i}\right)=0
$$

Noting that $\mathscr{B}$ is a countable subfamily of $\mathscr{C}$ completes the proof.

\section{REFERENCES}

1. E. O. Elliott, Measures on product spaces, Trans. Amer. Math. Soc. 128 (1967) 379-388.

2. E. O. Elliott and A. P. Morse, General product measures, Trans. Amer. Math. Soc. 110 (1964) 245-283.

3. M. Loeve, Probability theory, D. Van Nostrand Company, Princeton, New Jersey, 1960.

Received October 6, 1966, and in revised form February 3, 1969.

Bell Telephone Laboratories 


\section{PACIFIC JOURNAL OF MATHEMATICS}

\section{EDITORS}

H. ROYDEN

Stanford University

Stanford, California

\section{RichaRd PIERCE}

University of Washington Seattle, Washington 98105
J. DugundJI

Department of Mathematics

University of Southern California

Los Angeles, California 90007

BASIL GORDON

University of California

Los Angeles, California 90024

\section{ASSOCIATE EDITORS}
E. F. BECKENBACH
B. H. NeUMANN
F. WOLF
K. YOSHIDA

\section{SUPPORTING INSTITUTIONS}

UNIVERSITY OF BRITISH COLUMBIA CALIFORNIA INSTITUTE OF TECHNOLOGY

UNIVERSITY OF CALIFORNIA

MONTANA STATE UNIVERSITY

UNIVERSITY OF NEVADA

NEW MEXICO STATE UNIVERSITY

OREGON STATE UNIVERSITY

UNIVERSITY OF OREGON

OSAKA UNIVERSITY

UNIVERSITY OF SOUTHERN CALIFORNIA

\author{
STANFORD UNIVERSITY \\ UNIVERSITY OF TOKYO \\ UNIVERSITY OF UTAH \\ WASHINGTON STATE UNIVERSITY \\ UNIVERSITY OF WASHINGTON \\ AMERICAN MATHEMATICAL SOCIETY \\ CHEVRON RESEARCH CORPORATION \\ TRW SYSTEMS \\ NAVAL WEAPONS CENTER
}

The Supporting Institutions listed above contribute to the cost of publication of this Journal, but they are not owners or publishers and have no responsibility for its content or policies.

Mathematical papers intended for publication in the Pacific Journal of Mathematics should be in typed form or offset-reproduced, double spaced with large margins. Underline Greek letters in red, German in green, and script in blue. The first paragraph or two must be capable of being used separately as a synopsis of the entire paper. It should not contain references to the bibliography. Manuscripts, in duplicate if possible, may be sent to any one of the four editors. Please classify according to the scheme of Math. Rev. 36, 1539-1546. All other communications to the editors should be addressed to the managing editor, Richard Arens, University of California, Los Angeles, California, 90024.

50 reprints are provided free for each article; additional copies may be obtained at cost in multiples of 50 .

The Pacific Journal of Mathematics is published monthly. Effective with Volume 16 the price per volume (3 numbers) is $\$ 8.00$; single issues, $\$ 3.00$. Special price for current issues to individual faculty members of supporting institutions and to individual members of the American Mathematical Society: $\$ 4.00$ per volume; single issues $\$ 1.50$. Back numbers are available.

Subscriptions, orders for back numbers, and changes of address should be sent to Pacific Journal of Mathematics, 103 Highland Boulevard, Berkeley, California, 94708.

PUBLISHED BY PACIFIC JOURNAL OF MATHEMATICS, A NON-PROFIT CORPORATION

Printed at Kokusai Bunken Insatsusha (International Academic Printing Co., Ltd.), 7-17. Fujimi 2-chome, Chiyoda-ku, Tokyo, Japan. 


\section{Pacific Journal of Mathematics}

\section{Vol. 30, No. $3 \quad$ November, 1969}

Willard Ellis Baxter, Topological rings with property $(Y) \ldots \ldots \ldots \ldots . \ldots 5$

Sterling K. Berberian, Note on some spectral inequalities of $C . R$.

Putnam ..................................... 573

David Theodore Brown, Galois theory for Banach algebras . ........... 577

Dennis K. Burke and R. A. Stoltenberg, A note on p-spaces and Moore spaces ........................................ 601

Rafael Van Severen Chacon and Stephen Allan McGrath, Estimates of positive contractions....................................... 609

Rene Felix Dennemeyer, Conjugate surfaces for multiple integral problems in the calculus of variations ........................... 621

Edwin O. Elliott, Measures on countable product spaces.............. 639

John Moss Grover, Covering groups of groups of Lie type .............. 645

Charles Lemuel Hagopian, Concerning semi-local-connectedness and cutting in nonlocally connected continua .................. 657

Velmer B. Headley, A monotonicity principle for eigenvalues ........... 663

John Joseph Hutchinson, Intrinsic extensions of rings . . . . . . . . . . . . . 669

Harold H. Johnson, Determination of hyperbolicity by partial

prolongations .................................. 679

Tilla Weinstein, Holomorphic quadratic differentials on surfaces in $E^{3} \ldots 697$

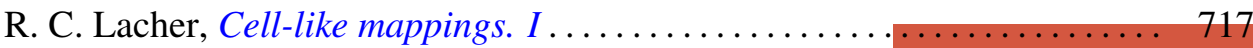

Roger McCann, A classification of centers

Curtis L. Outlaw, Mean value iteration of nonexpansive mappings in a

Banach space...

Allan C. Peterson, Distribution of zeros of solutions of a fourth order

differential equation.

Bhalchandra B. Phadke, Polyhedron inequality and strict convexity .. 765 Jack Wyndall Rogers Jr., On universal tree-like continua .

Edgar Andrews Rutter, Two characterizations of quasi-Frobenius rings

G. Sankaranarayanan and C. Suyambulingom, Some renewal theorems concerning a sequence of correlated random variables...

Joel E. Schneider, A note on the theory of primes........ . .

Richard Peter Stanley, Zero square rings .................

Edward D. Tymchatyn, The 2-cell as a partially ordered space

Craig A. Wood, On general Z.P.I.-rings................ 